\title{
Preoperative gene expression may be associated with neurocognitive decline after cardiopulmonary bypass
}

\author{
Ashraf A. Sabe, MD, ${ }^{a}$ Rahul S. Dalal, BS, ${ }^{\mathrm{a}}$ Louis M. Chu, MD, ${ }^{\mathrm{a}}$ Nassrene Y. Elmadhun, MD, ${ }^{\mathrm{a}}$ \\ Basel Ramlawi, MD, ${ }^{\mathrm{b}}$ Cesario Bianchi, MD, PhD, ${ }^{\mathrm{a}}$ and Frank W. Sellke, MD ${ }^{\mathrm{a}}$
}

Objective: Despite advances in surgical techniques, neurocognitive decline after cardiopulmonary bypass remains a common and serious complication. We have previously demonstrated that patients with neurocognitive decline have unique genetic responses 6 hours after cardiopulmonary bypass when compared with normal patients. We used genomic microarray to objectively investigate whether patients with neurocognitive decline had associated preoperative gene expression profiles and how these profiles changed up to 4 days after surgery.

\begin{abstract}
Methods: Patients undergoing cardiac surgery underwent neurocognitive assessments preoperatively and 4 days after surgery. Skeletal muscle was collected intraoperatively. Whole blood collected before cardiopulmonary bypass, 6 hours after cardiopulmonary bypass, and on postoperative day 4 was hybridized to Affymetrix Gene Chip U133 Plus 2.0 microarrays (Affymetrix Inc, Santa Clara, Calif). Gene expression in patients with neurocognitive decline was compared with gene expression in the normal group using JMP Genomics (SAS Institute Inc, Cary, NC). Only genes that were commonly expressed in the 2 groups with a false discovery rate of 0.05 and a fold change greater than 1.5 were carried forward to pathway analysis using Ingenuity Pathway Analysis (Ingenuity Systems, Redwood City, Calif). Microarray gene expression was validated by Green real-time polymerase chain reaction and Western blotting.

Results: Neurocognitive decline developed in 17 of 42 patients. A total of 54,675 common transcripts were identified on microarray in each group across all time points. Preoperatively, there were 140 genes that were significantly altered between the normal and neurocognitive decline groups $(P<.05)$. Pathway analysis demonstrated that preoperatively, patients with neurocognitive decline had increased regulation in genes associated with inflammation, cell death, and neurologic dysfunction. Of note, the number of significantly regulated genes between the 2 groups changed over each time point and decreased from 140 preoperatively to 64 six hours after cardiopulmonary bypass and to 25 four days after surgery. There was no correlation in gene expression between the blood and the skeletal muscle.
\end{abstract}

Conclusions: Patients in whom neurocognitive decline developed after cardiopulmonary bypass had increased differential gene expression before surgery versus patients in whom neurocognitive decline did not develop. Although significant differences in gene expression also existed postoperatively, these differences gradually decreased over time. Preoperative gene expression may be associated with neurologic injury after cardiopulmonary bypass. Further investigation into these genetic pathways may help predict patient outcome and guide patient selection. (J Thorac Cardiovasc Surg 2015;149:613-23)

See related commentary on pages 624-5.

From the Division of Cardiothoracic Surgery, ${ }^{\mathrm{a}}$ Cardiovascular Research Center, Warren Alpert Medical School of Brown University, Providence, RI; and Methodist DeBakey Heart \& Vascular Center, ${ }^{\text {b }}$ The Methodist Hospital, Houston, Tex.

Funding for this research was provided by the National Heart, Lung, and Blood Institute (HL46716 to F.W.S.) and National Institutes of Health Training Grant 5T32HL094300-03 (to A.A.S., L.M.C., and N.Y.E.).

Disclosures: Frank Sellke reports consulting fees from CSL Behring and the Medicines Company. All other authors have nothing to disclose with regard to commercial support.

Read at the 40th Annual Meeting of The Western Thoracic Surgical Association, Dana Point, California, June 25-28, 2014.

Received for publication June 25, 2014; revisions received Sept 29, 2014; accepted for publication Oct 9, 2014; available ahead of print Dec 4, 2014.

Address for reprints: Frank W. Sellke, MD, Division of Cardiothoracic Surgery, Cardiovascular Research Center, Warren Alpert Medical School of Brown University, 2 Dudley St, MOC 360, Providence, RI 02905 (E-mail: fsellke@lifespan.org). $0022-5223 / \$ 36.00$

Copyright $\odot 2015$ by The American Association for Thoracic Surgery

http://dx.doi.org/10.1016/j.jtcvs.2014.10.050
Neurocognitive dysfunction (NCD) is a common but poorly understood complication of cardiopulmonary bypass (CPB). Depending on the definition, as much as $80 \%$ of patients undergoing $\mathrm{CPB}$ may manifest neurologic complications postoperatively. ${ }^{1}$ Neurologic deficits are commonly divided into 2 categories: Type 1 deficits include focal neurologic events, such as stroke, stupor, and coma, and type 2 deficits are more global cognitive deficits, such as memory loss, confusion, and deterioration in intellectual function. ${ }^{2}$ Although type 1 deficits usually can be attributed to a specific cause, such as cerebral hypoperfusion or thromboembolic events, the cause of type 2 events is more vague. However, their incidence is similar to that of type 1 events, ${ }^{3}$ and they can be equally as devastating. A lack of understanding of the precipitating pathophysiology and inability to predict this type of injury only add to the strain on patients and their family members. 


\section{Abbreviations and Acronyms \\ $\mathrm{CPB}=$ cardiopulmonary bypass \\ $\mathrm{NCD}=$ neurocognitive dysfunction \\ $\mathrm{PCR}=$ polymerase chain reaction}

A variety of pathologic processes, including cerebral hypoperfusion, microembolization, inflammation, temperature changes, genetic predisposition, cerebral edema, or dysfunction of the blood-brain barrier, have been implicated in $\mathrm{NCD} .{ }^{4,5} \mathrm{CPB}$, although an essential component of the cardiac surgeon's armamentarium, has significant deleterious effects on the human body related to the interaction of blood components with the artificial surfaces of the circuit, including activation of leukocytes, cytokine release, and increase in reactive oxygen species. Our group, as well as others, previously demonstrated the association between systemic inflammation and NCD after CPB.${ }^{6,7}$ However, a comprehensive understanding of the precipitating and predisposing causes of NCD remains elusive, making accurate diagnosis and treatment difficult.

High-throughput microarray analysis provides insight into the response of nearly the entire human genome to a particular disease, and thus is an intriguing technique for identifying regulatory pathways and genes involved in poorly understood disease processes. Microarray technology has progressed exponentially in the past decade with the completion of the human genome project, development of more comprehensive microchips, and introduction of powerful pathway analysis software. We previously used microarray methods to show that genes associated with inflammation, antigen presentation, and cellular adhesion were differentially regulated in patients exhibiting NCD after CPB. In this prior study, same-group comparisons were made both in patients with NCD pre- and postoperatively and in normal patients pre- and postoperatively. ${ }^{8}$ We now compare normal patients with those with NCD pre- and postoperatively to assess whether there are inherent differences preoperatively leading to differential gene regulation 6 hours and 4 days post-CPB. The present study uses up-to-date microarray analytic techniques to identify specific cellular functions that may be involved in the development of NCD immediately and 4 days post-CPB.

\section{MATERIALS AND METHODS \\ Patient Enrollment}

We enrolled 43 patients scheduled electively or urgently for coronary artery bypass grafting, valvular surgery (aortic or mitral), or a combination of the 2 requiring CPB in this single-institution (Beth Israel Deaconess Medical Center, Boston, Mass) prospective cohort study. All forms and procedures were approved by the Beth Israel Deaconess Medical Center Institutional Review Board/Committee on Clinical Investigations. Preoperative informed consent was obtained from all study participants for surgical procedures performed and additional blood and tissue collection for the purpose of this investigation. Exclusion criteria included patients undergoing aortic arch/root procedures, patients with known calcified aortas or high-grade carotid stenosis, and patients with recent stroke, severe neurologic deficits, hepatic cirrhosis, or chronic renal failure (serum creatinine $>2.0 \mathrm{mg} / \mathrm{dL}$ ). Patients who were unable to complete baseline psychologic testing because of severe cognitive impairment, psychiatric disease, substance abuse, blindness, or poor English were also excluded. One enrolled patient was excluded because of the inability to complete the neuropsychologic assessment before discharge. Ultimately, 42 patients were included in the analysis.

\section{Surgical Technique}

All operations followed the conventional approach at our institution with regard to induction of general anesthesia, invasive monitoring, midline sternotomy, and systemic heparinization. CPB was initiated via right atrial and ascending aorta cannulae with a nonpulsatile system, membrane oxygenator, and $40-\mu \mathrm{m}$ arterial filter. Crystalloid pump prime was used. In all cases, mild hypothermic $\mathrm{CPB}\left(32^{\circ} \mathrm{C}-34^{\circ} \mathrm{C}\right)$ with intermittent cold blood hyperkalemic $(25 \mathrm{mmol} / \mathrm{L})$ cardioplegia was used. Serum glucose levels were monitored, and intermittent intravenous insulin injection or insulin infusion was used to target a level of less than $130 \mathrm{mg} / \mathrm{dL}$. While on $\mathrm{CPB}$, pump flow was maintained at 2 to $2.4 \mathrm{~L} / \mathrm{min} / \mathrm{m}^{2}$ body surface area. Arterial partial oxygen pressure was maintained between 150 and $250 \mathrm{~mm} \mathrm{Hg}$. Mean blood pressure was maintained between 50 and $90 \mathrm{~mm} \mathrm{Hg}$ by using conventional vasoactive medications.

\section{Neurocognitive Assessment}

Patients underwent evaluation with a battery of neurocognitive tests preoperatively (1-10 days before surgical intervention), on postoperative day 4 , and at 3 months postoperatively. All patients also underwent depression assessment with the Geriatric Depression Scale. All evaluations were carried out by trained, blinded psychometricians. ${ }^{8}$ Validated tools were used to assess memory, executive function, attention, language, and global cognition.

The Hopkins Verbal Learning Test assessed the number of items learned, the number of items recalled after a 20 -minute delay divided by the maximum number of items learned, and the number of items correctly identified from a list to measure verbal learning, retention, and recall. The Boston Naming Test was used to measure confrontational naming. ${ }^{9}$ Attention shifting ability was measured by recording time to complete the Trail Making A and B test. Digit Span was used to measure working memory and sustained attention span. Fluency was assessed by requiring patients to generate words in a category (semantic fluency) or beginning with a specific letter (phonemic fluency). The Wechsler Test of Adult Reading was used as a test of premorbid intelligence. The Stroop Color-Word Inference Test was used to assess executive function, and the Visual Search and Acuity Test assessed visuospatial abilities and executive function.

Patients with NCD were defined as those who demonstrated a 1 standard deviation decline from baseline on $25 \%$ of the tasks ( $2 / 8$ measures), in accordance with the "Statement of Consensus on Assessment of Neurobehavioral Outcomes After Cardiac Surgery." 10

\section{Sample Collection and Microarray Processing}

For all 42 patients, blood samples were collected from a central venous line preoperatively after induction of anesthesia and before skin incision (pre-CPB), early or 6 hours post-CPB in the intensive care unit, and late or 4 days post-CPB. Blood was drawn directly into PAXgene tubes (Qiagen Inc, Valencia, Calif) for mRNA stabilization and extraction, per the manufacturer's recommendation. Skeletal muscle samples were collected from 20 patients from the left intercostal muscle bed after cannulation but before the initiation of $\mathrm{CPB}$, and again after removal of the aortic 
crossclamp and weaning from CPB. Skeletal muscle samples were snapfrozen in liquid nitrogen immediately after collection and stored at $-80^{\circ} \mathrm{C}$.

RNA extraction and purification, cDNA synthesis, and production of biotin-labeled cRNA were completed by the Beth Israel Deaconess Medical Center Proteomics Core according to previously described protocols. ${ }^{11,12}$ cRNA from all samples were hybridized with Affymetrix GeneChip HG-U133 Plus 2.0 (Affymetrix Inc, Santa Clara, Calif), which probes for more than 38,500 genes. Chips were scanned with an HP G2500A ChipScanner (Affymetrix Inc), and low-level quality-control analysis and signal value measurement were performed using dChip software (Wong et al, Boston, Mass).$^{13}$ No outliers were identified by dChip, so all samples were carried on for subsequent analysis.

\section{Gene Expression and Pathway Analysis}

Gene expression analysis was performed on raw microchip data using JMP Genomics 4.0 (SAS Institute Inc, Cary, NC) for quality control, normalization, and statistical analysis. Composite chip data were normalized and compared using the Robust Multichip Average method, which revealed 1 blood and 1 skeletal muscle sample to be outliers. These were excluded from subsequent analysis. Gene expression in pre-CPB and post-CPB skeletal muscle samples and pre-CPB, 6 hours post-CPB, and 4 days post-CPB blood samples in patients with NCD were compared with the corresponding samples in patients without NCD using 1-way analysis of variance. A post hoc false detection rate algorithm with an alpha of 0.05 was applied to control for false-positives. Genes that were considered significantly regulated met 2 criteria: (1) mean fold change greater than 1.5 or less than -1.5 in patients with NCD compared with normal patients, and (2) $-\log (P$ value $)$ exceeding threshold calculated by the software for each comparison. All significant genes were uploaded into Ingenuity Pathway Analysis (Ingenuity Systems, Redwood City, Calif), which was used to generate the top canonical pathways involving the differentially regulated genes.

\section{Real-Time Polymerase Chain Reaction}

Gene expression analysis of whole blood-derived mRNA with HGU 133 Plus 2.0 chips was previously validated by real-time polymerase chain reaction (PCR). ${ }^{8}$ We used real-time PCR to validate gene expression analysis of skeletal muscle-derived mRNA. Total RNA was extracted from frozen sections of skeletal muscle using a Trizol-based method following the manufacturer's recommendations (Gibco BRL, Rockville, Md).

\section{RESULTS}

\section{Patient Characteristics}

As previously reported, early NCD developed at postoperative day 4 in 17 of the 42 patients included for analysis. After 3 months, all but 1 patient returned to their normal cognitive function. ${ }^{8}$ As demonstrated in our prior article, patients had similar baseline preoperative characteristics, including age, race, sex, and comorbidities. Likewise, patients' intraoperative course was well matched, including the type of procedure, time on CPB, crossclamp time, use of cell saver, and cardiotomy suction. Moreover, there were no differences in observed postoperative complications between the 2 groups, and there were no documented focal neurologic deficits or cerebrovascular events in any of the enrolled patients during this study period. ${ }^{8}$

\section{Gene Expression and Confirmation}

We have previously published a comprehensive database of gene expression in patients with and without NCD after

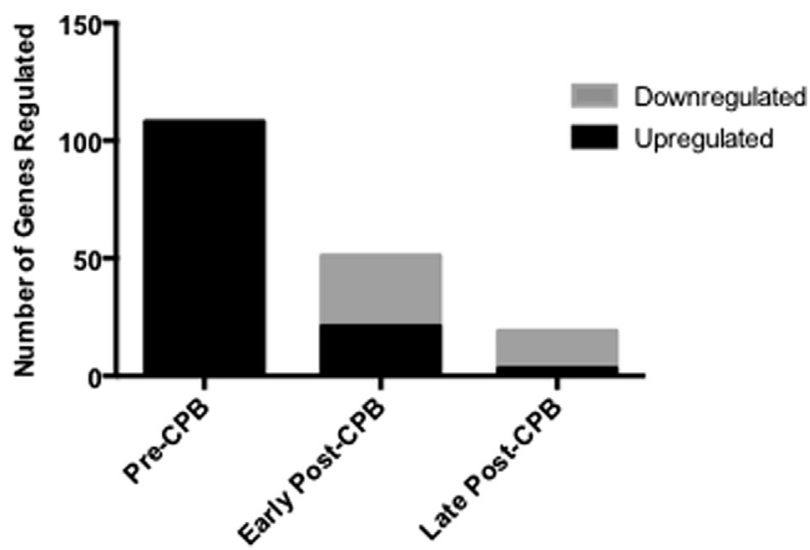

FIGURE 1. Number of genes significantly regulated in patients with NCD versus normal patients post-CPB. These represent named genes on pathway analysis. Early post-CPB: represents gene expression 6 hours post-CPB; late post-CPB: represents gene expression 4 days post-CPB. $C P B$, Cardiopulmonary bypass.

$\mathrm{CPB}$, including unsupervised hierarchical clustering of samples and confirmation of microarray gene expression with real-time PCR. ${ }^{8}$ A total of 54,675 transcripts were identified using our described microarray GeneChip.

\section{Preoperative Gene Expression and Pathway Analysis in Patients With Neurocognitive Dysfunction Compared With Normal Patients}

Preoperatively, there were 140 genes that were significantly altered between the normal and NCD groups, of which 108 were named. Of note, all 108 of these genes were upregulated in patients with NCD compared with normal patients (Figure 1 and Table 1). Pathway analysis was used to group genes by potential pathophysiologic function. This analysis demonstrated that preoperatively, patients with NCD had a significant increase in several genes involved in inflammation, cell death, and neurologic dysfunction. Selected genes have been listed in Table 2 . Gene expression in the blood was not correlated with gene expression in the skeletal muscle obtained at the time of surgery.

\section{Postoperative Gene Expression and Pathway Analysis in Patients With Neurocognitive Dysfunction Compared With Normal Patients}

Early postoperatively (6 hours), the number of significantly regulated genes decreased to 64 compared with preoperative gene regulation, of which 51 were named. A total of 21 of these 51 genes were significantly upregulated, whereas 30 were downregulated in patients with NCD compared with normal patients (Figure 1 and Table 3). Although the selected genes regulated were different than those regulated preoperatively, pathway analysis demonstrated regulation in several genes associated with 
TABLE 1. Preoperative gene expression in patients with neurocognitive dysfunction compared with normal patients: Complete list

\begin{tabular}{|c|c|c|c|c|c|}
\hline Accession ID & Gene name & FC & LCI & UCI & $P$ value \\
\hline \multicolumn{6}{|l|}{ Upregulated } \\
\hline$A D A$ & adenosine deaminase & 1.54 & 1.14 & 2.08 & .0059 \\
\hline ANAPC2 & anaphase promoting complex subunit 2 & 1.53 & 1.12 & 2.08 & .0081 \\
\hline АРОВЕCЗC & apolipoprotein B mRNA editing enzyme, catalytic polypeptide-like 3C & 1.73 & 1.24 & 2.40 & .0015 \\
\hline ARHGEF12 & Rho guanine nucleotide exchange factor (GEF) 12 & 1.63 & 1.19 & 2.22 & .0027 \\
\hline$A R H G E F 2$ & Rho/Rac guanine nucleotide exchange factor (GEF) 2 & 1.73 & 1.21 & 2.46 & .0031 \\
\hline ARID1A & AT rich interactive domain 1A (SWI-like) & 1.59 & 1.24 & 2.04 & .0004 \\
\hline ARID2 & AT rich interactive domain 2 (ARID, RFX-like) & 1.55 & 1.14 & 2.11 & .0060 \\
\hline$A R L A C$ & ADP-ribosylation factor-like $4 \mathrm{C}$ & 1.51 & 1.15 & 1.99 & .0040 \\
\hline$A S B 8$ & ankyrin repeat and SOCS box containing 8 & 1.73 & 1.23 & 2.43 & .0022 \\
\hline$A S X L 2$ & additional sex combs like 2 (Drosophila) & 1.55 & 1.15 & 2.08 & .0049 \\
\hline$A T P 2 B 4$ & ATPase, $\mathrm{Ca}++$ transporting, plasma membrane 4 & 1.72 & 1.21 & 2.44 & .0032 \\
\hline$B P T F$ & bromodomain PHD finger transcription factor & 1.68 & 1.16 & 2.43 & .0073 \\
\hline$C B L$ & $\mathrm{Cb} 1$ proto-oncogene, E3 ubiquitin protein ligase & 1.65 & 1.19 & 2.29 & .0034 \\
\hline$C D 3 E$ & CD3e molecule, epsilon (CD3-TCR complex) & 1.85 & 1.30 & 2.63 & .0009 \\
\hline$C D 3 G$ & CD3g molecule, gamma (CD3-TCR complex) & 1.77 & 1.28 & 2.44 & .0008 \\
\hline CIZ1 & CDKN1A interacting zinc finger protein 1 & 1.57 & 1.20 & 2.05 & .0014 \\
\hline CNPPD1 & cyclin Pas1/PHO80 domain containing 1 & 2.02 & & & .0033 \\
\hline$C T S B$ & cathepsin B & 2.44 & 1.43 & 4.16 & .0015 \\
\hline$D C A F 12$ & DDB1 and CUL4 associated factor 12 & 2.48 & 1.46 & 4.23 & .0011 \\
\hline$E 2 F 2$ & E2F transcription factor 2 & 1.66 & 1.23 & 2.25 & .0014 \\
\hline$E I F 2 A K 1$ & eukaryotic translation initiation factor 2-alpha kinase 1 & 2.25 & 1.25 & 4.03 & .0075 \\
\hline ELOF1 & elongation factor 1 homolog $(S$ cerevisiae $)$ & 1.57 & 1.13 & 2.18 & .0080 \\
\hline EML3 & echinoderm microtubule associated protein like 3 & 1.51 & 1.21 & 1.87 & .0004 \\
\hline$E P B 41$ & erythrocyte membrane protein band 4.1 (elliptocytosis $1, \mathrm{RH}$-linked) & 2.07 & 1.30 & 3.30 & .0026 \\
\hline FAM104A & family with sequence similarity 104 , member A & 2.20 & 1.43 & 3.38 & .0006 \\
\hline FAM117A & family with sequence similarity 117 , member A & 1.86 & 1.20 & 2.89 & .0068 \\
\hline FAM134A & family with sequence similarity 134 , member A & 1.62 & 1.14 & 2.30 & .0083 \\
\hline FAM46C & family with sequence similarity 46 , member C & 2.62 & 1.31 & 5.26 & .0074 \\
\hline$F A X D C 2$ & fatty acid hydroxylase domain containing 2 & 1.67 & & & .0044 \\
\hline FBXO9 & F-box protein 9 & 3.05 & 1.77 & 5.26 & .0001 \\
\hline FECH & ferrochelatase & 2.81 & 1.42 & 5.56 & .0036 \\
\hline$F K B P 1 B$ & FK506 binding protein $1 \mathrm{~B}, 12.6 \mathrm{kDa}$ & 2.65 & 1.75 & 4.03 & .0000 \\
\hline FOXO3 & forkhead box $\mathrm{O} 3$ & 1.99 & 1.26 & 3.15 & .0038 \\
\hline FTO & fat mass and obesity associated & 1.63 & 1.15 & 2.32 & .0069 \\
\hline FUNDC2 & FUN14 domain containing 2 & 1.86 & 1.20 & 2.87 & .0059 \\
\hline GDE1 (includes EG:393213) & glycerophosphodiester phosphodiesterase 1 & 2.06 & 1.29 & 3.28 & .0030 \\
\hline GSPT1 & G1 to $S$ phase transition 1 & 3.16 & 1.51 & 6.61 & .0041 \\
\hline$H B Z$ & hemoglobin, zeta & 1.72 & 1.16 & 2.56 & .0082 \\
\hline$H E C A$ & headcase homolog (Drosophila) & 1.50 & 1.12 & 2.02 & .0079 \\
\hline HECTD3 & HECT domain containing E3 ubiquitin protein ligase 3 & 1.89 & 1.46 & 2.45 & .0000 \\
\hline$H E M G N$ & hemogen & 2.04 & 1.23 & 3.38 & .0066 \\
\hline IBA57 & IBA57, iron-sulfur cluster assembly homolog ( $S$ cerevisiae $)$ & 1.69 & & & .0007 \\
\hline$I L 2 R G$ & interleukin 2 receptor, gamma & 1.96 & 1.23 & 3.14 & .0058 \\
\hline IL32 & interleukin 32 & 2.06 & 1.29 & 3.30 & .0032 \\
\hline ITM2A & integral membrane protein $2 \mathrm{~A}$ & 2.05 & 1.30 & 3.23 & .0027 \\
\hline$J H D M 1 D$ & jumonji $\mathrm{C}$ domain containing histone demethylase 1 homolog $\mathrm{D}$ ( $S$ cerevisiae $)$ & 1.74 & 1.17 & 2.59 & .0067 \\
\hline$J U N D$ & jun D proto-oncogene & 1.57 & 1.18 & 2.10 & .0024 \\
\hline KIAA1143 & KIAA1143 & 1.78 & 1.22 & 2.59 & .0032 \\
\hline KIAA1919 & KIAA1919 & 1.90 & 1.36 & 2.64 & .0003 \\
\hline KPNA1 & karyopherin alpha 1 (importin alpha 5) & 1.54 & 1.16 & 2.06 & .0037 \\
\hline KPNA6 & karyopherin alpha 6 (importin alpha 7) & 1.51 & 1.15 & 1.99 & .0041 \\
\hline MARCH8 & membrane-associated ring finger $(\mathrm{C} 3 \mathrm{HC} 4) 8$, E3 ubiquitin protein ligase & 2.52 & 1.43 & 4.43 & .0030 \\
\hline MINK1 & misshapen-like kinase 1 & 1.56 & 1.20 & 2.02 & .0013 \\
\hline MKRN1 & makorin ring finger protein 1 & 2.26 & 1.30 & 3.93 & .0046 \\
\hline MPHOSPH9 & M-phase phosphoprotein 9 & 1.51 & 1.20 & 1.90 & .0006 \\
\hline
\end{tabular}


TABLE 1. Continued

\begin{tabular}{|c|c|c|c|c|c|}
\hline Accession ID & Gene name & FC & LCI & UCI & $P$ value \\
\hline NDUFV3 & NADH dehydrogenase (ubiquinone) flavoprotein $3,10 \mathrm{kDa}$ & 1.53 & 1.12 & 2.07 & .0076 \\
\hline NFATC2 & nuclear factor of activated T-cells, cytoplasmic, calcineurin-dependent 2 & 1.83 & 1.20 & 2.79 & .0058 \\
\hline NTAN1 & $\mathrm{N}$-terminal asparagine amidase & 1.94 & 1.27 & 2.97 & .0029 \\
\hline OLA1 & Obg-like ATPase 1 & 1.83 & 1.26 & 2.64 & .0018 \\
\hline PAQR8 & progestin and adipoQ receptor family member VIII & 1.58 & 1.20 & 2.09 & .0015 \\
\hline PCGF5 & polycomb group ring finger 5 & 1.77 & 1.17 & 2.67 & .0081 \\
\hline PCSK5 & proprotein convertase subtilisin/kexin type 5 & 1.52 & 1.13 & 2.05 & .0066 \\
\hline$P I P 4 K 2 A$ & phosphatidylinositol-5-phosphate 4-kinase, type II, alpha & 2.23 & 1.28 & 3.88 & .0055 \\
\hline PITHD1 & $\begin{array}{l}\text { PITH (C-terminal proteasome-interacting domain of thioredoxin-like) domain } \\
\text { containing } 1\end{array}$ & 2.33 & & & .0011 \\
\hline PNISR & PNN-interacting serine/arginine-rich protein & 1.85 & & & .0030 \\
\hline$P R D X 2$ & peroxiredoxin 2 & 2.41 & 1.34 & 4.31 & .0039 \\
\hline PSME4 & proteasome (prosome, macropain) activator subunit 4 & 1.98 & 1.25 & 3.13 & .0043 \\
\hline PSMF1 & proteasome (prosome, macropain) inhibitor subunit 1 (PI31) & 1.82 & 1.22 & 2.72 & .0043 \\
\hline PTPN4 & protein tyrosine phosphatase, non-receptor type 4 (megakaryocyte) & 1.57 & 1.15 & 2.14 & .0054 \\
\hline$R A B 2 B$ & RAB2B, member RAS oncogene family & 2.95 & 1.72 & 5.08 & .0002 \\
\hline$R A L G D S$ & ral guanine nucleotide dissociation stimulator & 1.50 & 1.13 & 2.00 & .0064 \\
\hline RAPGEF6 & Rap guanine nucleotide exchange factor (GEF) 6 & 1.89 & 1.30 & 2.75 & .0011 \\
\hline$R G C C$ & regulator of cell cycle & 2.11 & & & .0021 \\
\hline RNF10 & ring finger protein 10 & 2.41 & 1.24 & 3.43 & .0059 \\
\hline$R N F 123$ & ring finger protein 123 & 2.03 & 1.25 & 3.31 & .0052 \\
\hline$R U N D C 3 A$ & RUN domain containing 3A & 1.95 & 1.34 & 2.82 & .0006 \\
\hline SCMLA & sex comb on midleg-like 4 (Drosophila) & 1.67 & 1.15 & 2.43 & .0082 \\
\hline SEC16A & SEC16 homolog A (S cerevisiae) & 1.86 & 1.20 & 2.89 & .0069 \\
\hline$S E C I S B P 2$ & SECIS binding protein 2 & 1.97 & 1.21 & 3.20 & .0070 \\
\hline SEPT6 & septin 6 & 1.50 & 1.14 & 1.97 & .0040 \\
\hline SESN3 & sestrin 3 & 2.25 & 1.32 & 3.84 & .0035 \\
\hline$S F 3 A 2$ & splicing factor $3 \mathrm{a}$, subunit $2,66 \mathrm{kDa}$ & 2.03 & 1.46 & 2.84 & .0001 \\
\hline SLC25A37 & solute carrier family 25 (mitochondrial iron transporter), member 37 & 2.50 & 1.37 & 4.55 & .0034 \\
\hline SLC38A5 & solute carrier family 38 , member 5 & 1.57 & 1.15 & 2.14 & .0055 \\
\hline SLC41A1 & solute carrier family 41 , member 1 & 1.51 & 1.32 & 1.73 & .0000 \\
\hline SLC48A1 & solute carrier family 48 (heme transporter), member 1 & 1.63 & 1.32 & 2.03 & .0000 \\
\hline SLC6A8 & solute carrier family 6 (neurotransmitter transporter, creatine), member 8 & 2.08 & 1.22 & 3.54 & .0080 \\
\hline SNAP29 & synaptosomal-associated protein, $29 \mathrm{kDa}$ & 1.59 & 1.22 & 2.08 & .0009 \\
\hline SNCA & synuclein, alpha (non A4 component of amyloid precursor) & 2.01 & 1.22 & 3.30 & .0068 \\
\hline SPTAN1 & spectrin, alpha, non-erythrocytic 1 & 2.05 & 1.30 & 3.24 & .0026 \\
\hline SSBP3 & single stranded DNA binding protein 3 & 1.62 & 1.18 & 2.22 & .0036 \\
\hline ST6GALNAC4 & $\begin{array}{l}\text { ST6 (alpha-N-acetyl-neuraminyl-2,3-beta-galactosyl-1,3)-N-acetylgalactosaminide } \\
\text { alpha-2,6-sialyltransferase } 4\end{array}$ & 1.74 & 1.24 & 2.45 & .0020 \\
\hline STAT4 & signal transducer and activator of transcription 4 & 1.71 & 1.17 & 2.51 & .0062 \\
\hline SUN2 & Sad 1 and UNC84 domain containing 2 & 1.63 & & & .0024 \\
\hline TMEM 245 & transmembrane protein 245 & 1.93 & & & .0031 \\
\hline TMEM86B & transmembrane protein $86 \mathrm{~B}$ & 1.56 & 1.14 & 2.14 & .0068 \\
\hline TMOD1 & tropomodulin 1 & 1.75 & 1.18 & 2.58 & .0059 \\
\hline TNS1 & tensin 1 & 3.03 & 1.53 & 6.03 & .0033 \\
\hline TOLLIP & toll interacting protein & 1.74 & 1.17 & 2.59 & .0070 \\
\hline$T P G S 2$ & tubulin polyglutamylase complex subunit 2 & 2.02 & & & .0068 \\
\hline TRIM58 & tripartite motif containing 58 & 2.49 & 1.35 & 4.60 & .0041 \\
\hline TSPAN5 & tetraspanin 5 & 2.74 & 1.57 & 4.76 & .0006 \\
\hline$T U B B 2 A$ & tubulin, beta $2 \mathrm{~A}$ class IIa & 4.58 & 1.64 & 12.80 & .0041 \\
\hline WDR26 & WD repeat domain 26 & 2.44 & 1.46 & 4.10 & .0010 \\
\hline WDR45 & WD repeat domain 45 & 1.89 & 1.22 & 2.87 & .0048 \\
\hline WNK1 & WNK lysine deficient protein kinase 1 & 1.92 & 1.22 & 3.02 & .0056 \\
\hline$Y Y 1$ & YY1 transcription factor & 1.97 & 1.31 & 2.97 & .0015 \\
\hline ZMAT2 & zinc finger, matrin-type 2 & 1.82 & 1.20 & 2.76 & .0058 \\
\hline
\end{tabular}

Gene expression listed as fold change in patients with NCD compared with normal patients. All values represented are significant $(P<.05)$. FC, Fold change; $L C I$, lower confidence interval; $U C I$, upper confidence interval. 
TABLE 2. Preoperative gene expression exhibiting significant regulation in patients with neurocognitive dysfunction compared with normal patients: Selected genes grouped by potential pathophysiologic function

\begin{tabular}{|c|c|c|c|c|c|}
\hline Accession ID & Gene name & FC & LCI & UCI & $P$ value \\
\hline \multicolumn{6}{|l|}{ Inflammation } \\
\hline$A D A$ & adenosine deaminase & 1.54 & 1.14 & 2.08 & .0059 \\
\hline$C D 3 E$ & CD3e molecule, epsilon (CD3-TCR complex) & 1.85 & 1.30 & 2.63 & .0009 \\
\hline$C D 3 G$ & CD3g molecule, gamma (CD3-TCR complex) & 1.77 & 1.28 & 2.44 & .0008 \\
\hline$I L 2 R G$ & interleukin 2 receptor, gamma & 1.96 & 1.23 & 3.14 & .0058 \\
\hline IL32 & interleukin 32 & 2.06 & 1.29 & 3.30 & .0032 \\
\hline NFATC2 & nuclear factor of activated T-cells, cytoplasmic, calcineurin-dependent 2 & 1.83 & 1.20 & 2.79 & .0058 \\
\hline STAT4 & signal transducer and activator of transcription 4 & 1.71 & 1.17 & 2.51 & .0062 \\
\hline \multicolumn{6}{|l|}{ Cell death } \\
\hline$C T S B$ & cathepsin B & 2.44 & 1.43 & 4.16 & .0015 \\
\hline$E 2 F 2$ & E2F transcription factor 2 & 1.66 & 1.23 & 2.25 & .0014 \\
\hline$E I F 2 A K 1$ & eukaryotic translation initiation factor 2-alpha kinase 1 & 2.25 & 1.25 & 4.03 & .0075 \\
\hline FOXO3 & forkhead box $\mathrm{O} 3$ & 1.99 & 1.26 & 3.15 & .0038 \\
\hline \multicolumn{6}{|c|}{ Neurologic dysfunction } \\
\hline$S N C A$ & synuclein, alpha (non A4 component of amyloid precursor) & 2.01 & 1.22 & 3.30 & .0068 \\
\hline FTO & fat mass and obesity associated & 1.63 & 1.15 & 2.32 & .0069 \\
\hline$T U B B 2 A$ & tubulin, beta $2 \mathrm{~A}$ class IIa & 4.58 & 1.64 & 12.80 & .0041 \\
\hline YY1 & YY1 transcription factor & 1.97 & 1.31 & 2.97 & .0015 \\
\hline SNAP29 & synaptosomal-associated protein, $29 \mathrm{kDa}$ & 1.59 & 1.22 & 2.08 & .0009 \\
\hline
\end{tabular}

Gene expression listed as fold change in patients with $\mathrm{NCD}$ compared with normal patients. All values represented are significant $(P<.05) . F C$, Fold change; $L C I$, lower confidence interval; $U C I$, upper confidence interval.

inflammation, cell death, and neurologic dysfunction in patients with NCD compared with normal patients (Table 4). Late postoperatively (4 days), the number of significantly regulated genes decreased to 25 , of which 19 were named (Figure 1 and Table 5). Three of these 19 genes were upregulated, and the remaining 16 genes were downregulated in patients with NCD compared with normal patients (Table 5). Selected genes involved with inflammation, cell death, and neurologic dysfunction are listed in Table 6, of which all were all actually downregulated in patients with NCD compared with normal patients.

\section{DISCUSSION}

The current study demonstrates that patients in whom NCD developed post-CPB have differential gene expression before surgery versus patients in whom NCD did not develop. Although significant differences in gene expression exist post-CPB, they decreased over time. These findings suggest that patients may be inherently predisposed to NCD after CPB independently of surgical or anesthetic technique. This notion is certainly supported by the failure to reduce the incidence of type $2 \mathrm{NCD}$, despite improvements in operative techniques. ${ }^{14}$ To improve these outcomes, novel diagnostic and therapeutic techniques will need to be used with a focus on identifying individual genetic variants associated with disease susceptibility and therapeutic response. The use of up-to-date microarray and bioinformatics analysis is an important step in beginning to address these challenges.
Pre-CPB, 108 named genes were significantly regulated in patients with NCD. Several genes involved with inflammation, cell death, and neurologic dysfunction were increased in patients in whom NCD would later develop. Systemic inflammation has been shown to contribute to neurocognitive decline after $\mathrm{CPB} .^{7,15,16}$ In a previous study, we demonstrated that although an increase in preoperative inflammatory chemokines did not affect outcome, postoperative elevations in chemokines were associated with the development of delirium after $\mathrm{CPB} .^{17}$ Chemokines act as potent immune mediators and may attract inflammatory cells, resulting in a disruption of the blood-brain barrier and cognitive dysfunction. In our current study, we demonstrate an elevation in several genes associated with T-cell activation and signaling preoperatively in patients in whom NCD would later develop. For instance, patients in whom NCD developed postoperatively had significantly elevated regulation in genes implicated in T-cell activation, maturation, and cytokine signaling, including ADA, CD3E, CD3G, IL2RG, IL32, NFATC2, and STAT4. ${ }^{18-20}$ Perhaps these inherent elevations result in accentuated inflammatory response and resultant increase in chemokine production. These patients also had a significant increase in genes associated with cell death and oxidative stress, such as E2F2, EIF2AK1, and FOX03. ${ }^{21-23}$ Furthermore, patients in whom NCD developed also had an increase in genes more directly associated with neurologic dysfunction, such as SNCA, FTO, TUBB2A, YY1, and SNAP29. ${ }^{24-28}$ Although these genes are not directly related to one another in a specific 
TABLE 3. Early postcardiopulmonary bypass ( 6 hours postcardiopulmonary bypass) gene expression in patients with neurocognitive dysfunction compared with normal patients: Complete list

\begin{tabular}{|c|c|c|c|c|c|}
\hline Accession ID & Gene name & FC & LCI & UCI & $P$ value \\
\hline \multicolumn{6}{|l|}{ Upregulated } \\
\hline$C B L$ & $\mathrm{Cbl}$ proto-oncogene, E3 ubiquitin protein ligase & 1.61 & 1.17 & 2.23 & .0046 \\
\hline CCNJL & cyclin J-like & 1.70 & 1.21 & 2.37 & .0025 \\
\hline CDK5RAP2 & CDK5 regulatory subunit associated protein 2 & 1.51 & 1.12 & 2.04 & .0074 \\
\hline CEP19 & centrosomal protein $19 \mathrm{kDa}$ & 1.50 & & & .0071 \\
\hline$C L E C 1 B$ & C-type lectin domain family 1 , member B & 1.56 & & & .0013 \\
\hline DACH1 & dachshund homolog 1 (Drosophila) & 1.74 & 1.33 & 2.28 & .0008 \\
\hline DHRS12 & dehydrogenase/reductase (SDR family) member 12 & 1.67 & 1.25 & 2.23 & .0002 \\
\hline EPAS1 & endothelial PAS domain protein 1 & 1.73 & 1.31 & 2.27 & .0039 \\
\hline$F K B P 1 B$ & FK506 binding protein $1 \mathrm{~B}, 12.6 \mathrm{kDa}$ & 2.04 & 1.35 & 3.09 & .0010 \\
\hline GPSM3 & G-protein signaling modulator 3 & 2.02 & 1.37 & 2.99 & .0006 \\
\hline GRB10 & growth factor receptor-bound protein 10 & 1.54 & 1.26 & 1.88 & .0001 \\
\hline KBTBD6 & kelch repeat and BTB (POZ) domain containing 6 & 1.78 & 1.27 & 2.48 & .0010 \\
\hline MARCH8 & membrane-associated ring finger $(\mathrm{C} 3 \mathrm{HC} 4) 8, \mathrm{E} 3$ ubiquitin protein ligase & 1.64 & 1.16 & 2.32 & .0058 \\
\hline METTL21D & methyltransferase-like protein $21 \mathrm{D}$ & 1.51 & & & .0032 \\
\hline$O L A H$ & oleoyl-ACP hydrolase & 1.88 & 1.41 & 2.50 & .0000 \\
\hline$O S B P 2$ & oxysterol binding protein 2 & 1.85 & 1.31 & 2.62 & .0008 \\
\hline$P D Z K 1 I P 1$ & PDZK1 interacting protein 1 & 2.37 & 1.32 & 4.24 & .0045 \\
\hline$T L R 4$ & toll-like receptor 4 & 1.78 & 1.18 & 2.67 & .0070 \\
\hline TPK1 & thiamin pyrophosphokinase 1 & 1.67 & 1.24 & 2.24 & .0011 \\
\hline TSPAN5 & tetraspanin 5 & 2.17 & 1.25 & 3.77 & .0065 \\
\hline ZBTB16 & zinc finger and BTB domain containing 16 & 1.94 & 1.32 & 2.86 & .0010 \\
\hline \multicolumn{6}{|l|}{ Downregulated } \\
\hline$A C A D M$ & acyl-CoA dehydrogenase, $\mathrm{C}-4$ to $\mathrm{C}-12$ straight chain & 0.58 & 0.40 & 0.85 & .0065 \\
\hline$A L G 13$ & ALG13, UDP-N-acetylglucosaminyltransferase subunit & 0.51 & 0.36 & 0.73 & .0005 \\
\hline ANKRD10 & ankyrin repeat domain 10 & 0.58 & 0.41 & 0.81 & .0021 \\
\hline$A R G L U 1$ & arginine and glutamate rich 1 & 0.39 & 0.20 & 0.76 & .0067 \\
\hline$A T F 1$ & activating transcription factor 1 & 0.63 & 0.45 & 0.88 & .0079 \\
\hline C2orf49 & chromosome 2 open reading frame 49 & 0.66 & 0.51 & 0.86 & .0025 \\
\hline CLIP4 & CAP-GLY domain containing linker protein family, member 4 & 0.65 & 0.48 & 0.88 & .0059 \\
\hline CPEB2 & cytoplasmic polyadenylation element binding protein 2 & 0.54 & 0.37 & 0.79 & .0020 \\
\hline CRISP2 & cysteine-rich secretory protein 2 & 0.63 & 0.50 & 0.79 & .0001 \\
\hline CSE1L & CSE1 chromosome segregation 1-like (yeast) & 0.64 & 0.48 & 0.86 & .0035 \\
\hline GPR84 & G protein-coupled receptor 84 & 0.53 & 0.34 & 0.83 & .0062 \\
\hline KANSL2 & KAT8 regulatory NSL complex subunit 2 & 0.64 & & & .0032 \\
\hline MALT1 & mucosa associated lymphoid tissue lymphoma translocation gene 1 & 0.63 & 0.51 & 0.78 & .0001 \\
\hline$M B N L 2$ & muscleblind-like splicing regulator 2 & 0.65 & 0.50 & 0.85 & .0017 \\
\hline$M I R 22 H G$ & MIR22 host gene (non-protein coding) & 0.62 & & & .0008 \\
\hline MON2 & MON2 homolog $(S$ cerevisiae $)$ & 0.63 & 0.47 & 0.84 & .0024 \\
\hline MPHOSPH6 & M-phase phosphoprotein 6 & 0.59 & 0.46 & 0.76 & .0001 \\
\hline NADSYN1 & NAD synthetase 1 & 0.60 & 0.42 & 0.85 & .0051 \\
\hline$P I K 3 C 2 A$ & phosphatidylinositol-4-phosphate 3-kinase, catalytic subunit type 2 alpha & 0.60 & 0.42 & 0.86 & .0057 \\
\hline RALGAPAl & Ral GTPase activating protein, alpha subunit 1 (catalytic) & 0.65 & & & .0015 \\
\hline$R N F 144 B$ & ring finger protein 144B & 0.66 & 0.51 & 0.84 & .0012 \\
\hline$R W D D 4$ & RWD domain containing 4 & 0.65 & 1.20 & 2.65 & .0013 \\
\hline SH3GL3 & SH3-domain GRB2-like 3 & 0.56 & 0.50 & 0.84 & .0045 \\
\hline SREK1 & splicing regulatory glutamine/lysine-rich protein 1 & 0.62 & & & .0011 \\
\hline$T A P 1$ & transporter 1, ATP-binding cassette, sub-family B (MDR/TAP) & 0.61 & 0.43 & 0.87 & .0075 \\
\hline TFEC & transcription factor EC & 0.62 & 0.45 & 0.84 & .0025 \\
\hline TMEM168 & transmembrane protein 168 & 0.66 & 0.52 & 0.84 & .0011 \\
\hline $\mathrm{ZCCHC2}$ & zinc finger, $\mathrm{CCHC}$ domain containing 2 & 0.57 & 0.39 & 0.82 & .0033 \\
\hline $\mathrm{ZCCHC8}$ & zinc finger, $\mathrm{CCHC}$ domain containing 8 & 0.65 & 0.49 & 0.86 & .0033 \\
\hline ZMYND11 & zinc finger, MYND-type containing 11 & 0.65 & 0.49 & 0.85 & .0027 \\
\hline
\end{tabular}

Gene expression listed as fold change in patients with NCD compared with normal patients. All values represented are significant $(P<.05)$. $F C$, Fold change; $L C I$, lower confidence interval; $U C I$, upper confidence interval. 
TABLE 4. Early postcardiopulmonary bypass ( 6 hours postcardiopulmonary bypass) gene expression in patients with neurocognitive dysfunction compared with normal patients: Selected genes grouped by potential pathophysiologic function

\begin{tabular}{|c|c|c|c|c|c|}
\hline Accession ID & Gene name & FC & LCI & UCI & $P$ value \\
\hline \multicolumn{6}{|l|}{ Inflammation } \\
\hline$C L E C 1 B$ & C-type lectin domain family 1 , member B & 1.56 & & & .0013 \\
\hline$T L R 4$ & toll-like receptor 4 & 1.78 & 1.18 & 2.67 & .0070 \\
\hline$T A P 1$ & transporter 1, ATP-binding cassette, sub-family B (MDR/TAP) & 0.61 & 0.43 & 0.87 & .0075 \\
\hline MALT1 & mucosa associated lymphoid tissue lymphoma translocation gene 1 & 0.63 & 0.51 & 0.78 & .0001 \\
\hline \multicolumn{6}{|l|}{ Cell death } \\
\hline EPAS1 & endothelial PAS domain protein 1 & 1.73 & 1.31 & 2.27 & .0002 \\
\hline ZBTB16 & zinc finger and BTB domain containing 16 & 1.94 & 1.32 & 2.86 & .0010 \\
\hline \multicolumn{6}{|c|}{ Neurologic dysfunction } \\
\hline CDK5RAP2 & CDK5 regulatory subunit associated protein 2 & 1.51 & 1.12 & 2.04 & .0074 \\
\hline
\end{tabular}

Gene expression listed as fold change in patients with NCD compared with normal patients. All values represented are significant $(P<.05) . F C$, Fold change; $L C I$, lower confidence interval; $U C I$, upper confidence interval.

TABLE 5. Late postcardiopulmonary bypass (4 days postcardiopulmonary bypass) gene expression in patients with neurocognitive dysfunction compared with normal patients: Complete list

\begin{tabular}{|c|c|c|c|c|c|}
\hline Accession ID & Gene name & FC & LCI & UCI & $\begin{array}{c}P \\
\text { value }\end{array}$ \\
\hline \multicolumn{6}{|l|}{ Upregulated } \\
\hline$D D X 17$ & $\begin{array}{l}\text { DEAD (Asp-Glu-Ala-Asp) box } \\
\text { helicase } 17\end{array}$ & 1.89 & 1.34 & 2.26 & .0001 \\
\hline MLLT10 & $\begin{array}{l}\text { myeloid/lymphoid or mixed- } \\
\text { lineage leukemia (trithorax } \\
\text { homolog, Drosophila); } \\
\text { translocated to, } 10\end{array}$ & 1.57 & 1.31 & 1.88 & .0000 \\
\hline$P G M 2 L 1$ & phosphoglucomutase 2-like 1 & 1.69 & 1.19 & 2.41 & .0042 \\
\hline \multicolumn{6}{|c|}{ Downregulated } \\
\hline C11orf31 & $\begin{array}{l}\text { chromosome } 11 \text { open reading } \\
\text { frame } 31\end{array}$ & 0.65 & 0.49 & 0.88 & .0057 \\
\hline$D I A B L O$ & $\begin{array}{l}\text { diablo, IAP-binding mitochondrial } \\
\text { protein }\end{array}$ & 0.61 & & & .0013 \\
\hline GIMAP4 & GTPase, IMAP family member 4 & 0.46 & 0.43 & 0.87 & .0067 \\
\hline GPR183 & $\mathrm{G}$ protein-coupled receptor 183 & 0.56 & 0.27 & 0.79 & .0059 \\
\hline HDAC9 & histone deacetylase 9 & 0.67 & 0.39 & 0.80 & .0021 \\
\hline MAT2A & $\begin{array}{l}\text { methionine adenosyltransferase II, } \\
\text { alpha }\end{array}$ & 0.64 & & & .0075 \\
\hline$M E F 2 C$ & myocyte enhancer factor $2 \mathrm{C}$ & 0.47 & 0.47 & 0.88 & .0075 \\
\hline PPIA & $\begin{array}{l}\text { peptidylprolyl isomerase A } \\
\text { (cyclophilin A) }\end{array}$ & 0.66 & 0.28 & 0.77 & .0060 \\
\hline PTMA & prothymosin, alpha & 0.56 & 0.38 & 0.83 & .0045 \\
\hline RPL10 & ribosomal protein L10 & 0.60 & 0.41 & 0.87 & .0073 \\
\hline RPL12 & ribosomal protein L12 & 0.60 & 0.41 & 0.87 & .0079 \\
\hline RPL15 & ribosomal protein L15 & 0.57 & 0.38 & 0.86 & .0078 \\
\hline RPS13 & ribosomal protein $\mathrm{S} 13$ & 0.56 & 0.39 & 0.81 & .0030 \\
\hline Sept9 & septin 9 & 0.62 & 0.44 & 0.87 & .0072 \\
\hline$S P C S 3$ & $\begin{array}{c}\text { signal peptidase complex subunit } \\
3 \text { homolog }(\text { S cerevisiae })\end{array}$ & 0.64 & 0.48 & 0.85 & .0031 \\
\hline$W W P 1$ & $\begin{array}{r}\text { WW domain containing E3 } \\
\text { ubiquitin protein ligase } 1\end{array}$ & 0.66 & 0.52 & 0.83 & .0008 \\
\hline
\end{tabular}

$\overline{\text { Gene expression listed as fold change in patients with NCD compared with normal }}$ patients. All values represented are significant $(P<.05)$. $F C$, Fold change; $L C I$, lower confidence interval; $U C I$, upper confidence interval. pathway, bioinformatics analysis demonstrates that they do share important roles in neurologic function and cognition. $S N C A$ is abundantly expressed in the brain and a major component of amyloid plaques in Alzheimer's disease. ${ }^{24}$ $F T O$, which has been shown to be inversely associated with brain volume, is also associated with Alzheimer's disease and reduced verbal fluency in obese patients. ${ }^{25,26}$ $T U B B 2 A$ is involved in microtubule and axonal guidance, and SNAP29 has been shown to mediate synaptic membrane docking and may slow neurotransmitter release. ${ }^{28}$ YYl has many roles in neuronal development and dysfunction and often plays a larger role in activating or repressing gene expression. ${ }^{27}$

There was a relative decrease in the number of genes regulated postoperatively when comparing patients with and without NCD. Again, these findings suggest that

TABLE 6. Late postcardiopulmonary bypass (4 days postcardiopulmonary bypass) gene expression in patients with neurocognitive dysfunction compared with normal patients: Selected genes grouped by potential pathophysiologic function

\begin{tabular}{|c|c|c|c|c|c|}
\hline Accession ID & Gene name & FC & LCI & UCI & $P$ value \\
\hline \multicolumn{6}{|l|}{ Inflammation } \\
\hline GIMAP4 & $\begin{array}{l}\text { GTPase, IMAP family } \\
\text { member } 4\end{array}$ & 0.45 & 0.27 & 0.79 & .0059 \\
\hline PTMA & prothymosin, alpha & 0.56 & 0.38 & 0.83 & .0045 \\
\hline GPR183 & $\begin{array}{l}\text { G protein-coupled } \\
\text { receptor } 183\end{array}$ & 0.56 & 0.39 & 0.80 & .0021 \\
\hline \multicolumn{6}{|l|}{ Cell death } \\
\hline$D I A B L O$ & $\begin{array}{l}\text { diablo, IAP-binding } \\
\text { mitochondrial protein }\end{array}$ & 0.61 & 0.43 & 0.87 & .0045 \\
\hline HDAC9 & Histone deacetylase 9 & 0.67 & & & .0075 \\
\hline \multicolumn{6}{|c|}{ Neurologic dysfunction } \\
\hline$M E F 2 C$ & $\begin{array}{l}\text { myocyte enhancer } \\
\text { factor } 2 \mathrm{C}\end{array}$ & 0.47 & 0.28 & 0.77 & .0039 \\
\hline
\end{tabular}

$\overline{\text { Gene expression listed as fold change in patients with NCD compared with normal }}$ patients. All values represented are significant $(P<.05)$. $F C$, Fold change; $L C I$, lower confidence interval; $U C I$, upper confidence interval. 
patients may be inherently predisposed to the development of NCD after CPB. Further investigations may reveal predictive patterns in gene expression and ultimately result in improved preoperative planning and care of patients undergoing cardiac surgery.

\section{Limitations and Future Directions}

There are limitations to this study. Although our baseline patient characteristics and operative techniques were similar in this single institution study, the number of patients in the study was limited. A larger sample of patients would help provide greater insight into the unique gene expression profiles associated with NCD and allow for a more extensive mapping of gene pathways, as opposed to just placing genes in functional groups, as we have done. Another limitation is that we did not directly sample brain tissue for our mRNA extraction. We could not biopsy brain tissue in patients, and even if this were done it would not be feasible as a regular diagnostic or screening tool in a clinical setting. Of note, many of the regulated genes, which have been discussed in this article, are associated with on inflammatory processes in the blood, which could secondarily affect the brain. We did sample skeletal muscle, which like brain tissue would not be exposed to cardioplegia but CPB alone; however, there were no correlations in gene regulation between the blood and muscle samples.

It is also important to note that this study would need to be repeated before any claim can be made as to whether the aforementioned genes were indeed predictive of NCD in patient populations. Although the results of this current study highly suggest that preoperative gene expression is associated with postoperative NCD, we must also be cautious with the interpretation of microarray. To demonstrate predictive gene expression patterns, another study would need to be designed with a new group of patients, in whom genes would be checked in a prospective manner preoperatively to determine whether any of the genes identified in the current study were actually a predictor of later NCD in new patient cohorts.

Another common pitfall with the interpretation of microarray is errors with the statistical treatment of the data. Because microarray identifies tens of thousands of individual genes, random chance alone can often result in significant $P$ values when simple statistical analysis is performed. To account for this potential error in false discovery, using specialized statistical software, we performed analysis of variance testing with multiple comparison correction and limited our false discovery rate to less than 0.05 . This is widely accepted as a stringent method to help prevent an error in multiple comparisons, and although it is not a universal application in the interpretation of microarray, it does improve the likely reproducibility of the results.

\section{CONCLUSIONS}

This work represents a follow-up study of a microarray database compiled in 2007. Although our prior study identified differences in gene expression after CPB in patients with and without NCD, the current study is the first to directly investigate the differences in genetic regulation of patients with NCD compared with normal patients pre- and post-CPB. Currently, these studies should serve primarily as a database to guide further genetic studies in different patient cohorts. The overarching goal of this project is to guide novel diagnostic techniques to help identify inherent genetic variations associated with susceptibility of disease, and ultimately to improve preoperative patient selection and individualized therapeutic techniques.

\section{References}

1. Gao L, Taha R, Gauvin D, Othmen LB, Wang Y, Blaise G. Postoperative cognitive dysfunction after cardiac surgery. Chest. 2005;128:3664-70.

2. Eagle KA, Guyton RA, Davidoff R, Edwards FH, Ewy GA, Gardner TJ, et al ACC/AHA 2004 guideline update for coronary artery bypass graft surgery: a report of the American College of Cardiology/American Heart Association Task Force on Practice Guidelines (Committee to Update the 1999 Guidelines for Coronary Artery Bypass Graft Surgery). Circulation. 2004;110:e340-437.

3. Roach GW, Kanchuger M, Mangano CM, Newman M, Nussmeier N, Wolman R, et al. Adverse cerebral outcomes after coronary bypass surgery. Multicenter Study of Perioperative Ischemia Research Group and the Ischemia Research and Education Foundation Investigators. N Engl J Med. 1996;335:1857-63.

4. Murkin JM. Etiology and incidence of brain dysfunction after cardiac surgery. $J$ Cardiothorac Vasc Anesthes. 1999;13:12-7; discussion 36-7.

5. Selnes OA, McKhann GM. Neurocognitive complications after coronary artery bypass surgery. Ann Neurol. 2005;57:615-21.

6. Baufreton C, Allain P, Chevailler A, Etcharry-Bouyx F, Corbeau JJ, Legall D, et al Brain injury and neuropsychological outcome after coronary artery surgery are affected by complement activation. Ann Thorac Surg. 2005;79:1597-605.

7. Ramlawi B, Rudolph JL, Mieno S, Feng J, Boodhwani M, Khabbaz K, et al. C-Reactive protein and inflammatory response associated to neurocognitive decline following cardiac surgery. Surgery. 2006;140:221-6.

8. Ramlawi B, Otu H, Rudolph JL, Mieno S, Kohane IS, Can H, et al. Genomic expression pathways associated with brain injury after cardiopulmonary bypass. J Thorac Cardiovasc Surg. 2007;134:996-1005.

9. Mack WJ, Freed DM, Williams BW, Henderson VW. Boston Naming Test: shortened versions for use in Alzheimer's disease. J Gerontol. 1992;47:P154-8.

10. Murkin JM, Newman SP, Stump DA, Blumenthal JA. Statement of consensus on assessment of neurobehavioral outcomes after cardiac surgery. Ann Thorac Surg. 1995;59:1289-95.

11. Jones J, Otu H, Spentzos D, Kolia S, Inan M, Beecken WD, et al. Gene signatures of progression and metastasis in renal cell cancer. Clin Cancer Res. 2005;11:5730-9.

12. Ruel M, Bianchi C, Khan TA, Xu S, Liddicoat JR, Voisine P, et al. Gene expression profile after cardiopulmonary bypass and cardioplegic arrest. J Thorac Cardiovasc Surg. 2003;126:1521-30.

13. Li C, Wong WH. Model-based analysis of oligonucleotide arrays: expression in dex computation and outlier detection. Proc Natl Acad Sci U S A. 2001;98:31-6.

14. Goto T, Maekawa K. Cerebral dysfunction after coronary artery bypass surgery. $J$ Anesth. 2014;28:242-8.

15. Jungwirth B, Kellermann K, Qing M, Mackensen GB, Blobner M, Kochs EF Cerebral tumor necrosis factor alpha expression and long-term neurocognitive performance after cardiopulmonary bypass in rats. J Thorac Cardiovasc Surg. 2009; 138:1002-7.

16. Hogan AM, Shipolini A, Brown MM, Hurley R, Cormack F. Fixing hearts and protecting minds: a review of the multiple, interacting factors influencing cognitive function after coronary artery bypass graft surgery. Circulation. 2013;128:162-71.

17. Rudolph JL, Ramlawi B, Kuchel GA, McElhaney JE, Xie D, Sellke FW, et al. Chemokines are associated with delirium after cardiac surgery. J Gerontol A Biol Sci Med Sci. 2008;63:184-9.

18. Martinez-Navio JM, Climent N, Gallart T, Lluis C, Franco R. An old enzyme for current needs: adenosine deaminase and a dendritic cell vaccine for HIV Immunol Cell Biol. 2012;90:594-600. 
19. Batista A, Millan J, Mittelbrunn M, Sanchez-Madrid F, Alonso MA. Recruitment of transferrin receptor to immunological synapse in response to TCR engagement. J Immunol. 2004;172:6709-14.

20. Park H, Li Z, Yang XO, Chang SH, Nurieva R, Wang YH, et al. A distinct lineage of CD4 T cells regulates tissue inflammation by producing interleukin 17. Nat Immunol. 2005;6:1133-41.

21. Muller H, Bracken AP, Vernell R, Moroni MC, Christians F, Grassilli E, et al. E2Fs regulate the expression of genes involved in differentiation, development, proliferation, and apoptosis. Genes Dev. 2001;15:267-85.

22. Liu S, Suragani RN, Wang F, Han A, Zhao W, Andrews NC, et al. The function of heme-regulated eIF2alpha kinase in murine iron homeostasis and macrophage maturation. J Clin Invest. 2007;117:3296-305.

23. Hagenbuchner J, Ausserlechner MJ. Mitochondria and FOXO3: breath or die. Front Physiol. 2013;4:147.

24. Van der Schyf CJ, Geldenhuys WJ, Youdim MB. Multifunctional drugs with different CNS targets for neuropsychiatric disorders. J Neurochem. 2006;99:1033-48.

25. Keller L, Xu W, Wang HX, Winblad B, Fratiglioni L, Graff C. The obesity related gene, FTO, interacts with APOE, and is associated with Alzheimer's disease risk: a prospective cohort study. J Alzheimers Dis. 2011;23:461-9.

26. Benedict C, Jacobsson JA, Ronnemaa E, Sallman-Almen M, Brooks S, Schultes B, et al. The fat mass and obesity gene is linked to reduced verbal fluency in overweight and obese elderly men. Neurobiol Aging. 2011;32:1159.e1-5.

27. He Y, Casaccia-Bonnefil P. The Yin and Yang of YY1 in the nervous system. J Neurochem. 2008;106:1493-502

28. Pan PY, Cai Q, Lin L, Lu PH, Duan S, Sheng ZH. SNAP-29-mediated modulation of synaptic transmission in cultured hippocampal neurons. J Biol Chem. 2005; 280:25769-79.

\section{Discussion}

Dr Sabe. Good morning, everyone. Thank you for the opportunity to present today.

I have no disclosures.

Neurocognitive dysfunction (NCD) is a poorly understood complication after cardiac surgery. As high as $70 \%$ of patients undergoing cardiopulmonary bypass (CPB) surgery manifest neurologic complications postoperatively. Neurologic dysfunction after surgery is commonly divided into 1 of 2 types. Type I usually has clear manifestations, as seen with stroke, stupor, or coma, and specific causes, including cerebral hypoperfusion or thromboembolic events. Type II deficits are often less clear, manifesting as memory loss, confusion, and deterioration of intellectual function. The causes of these deficits are unknown, but they have been theoretically attributed to a range of causes, including hypoperfusion, microembolization, inflammation, temperature changes, cerebral edema, and age. Over the past 20 years, despite advances in CPB, anesthesia, and surgical techniques, the incidence of these type II deficits has not significantly improved. The resultant in-hospital costs have been estimated to surpass $\$ 400$ million annually, and when postdischarge care is taken into account, those cost estimates range from $\$ 2$ to $\$ 4$ billion. The inability to predict type II events further burdens patients, their families, and their caretakers. We hypothesized that patients who develop type II NCD after cardiac surgery have inherent differences in gene expression preoperatively. We utilized microarray to study differential gene regulation preoperatively, 6 hours after surgery, and 4 days after surgery.

This prospective study was performed at a single institution. We enrolled adult patients undergoing cardiac surgery with $\mathrm{CPB}$. Patients were excluded if they required aortic arch or root procedures; or had significant calcified aortas, high-grade carotid stenosis; baseline neurologic, cognitive, or psychological impairments; cirrhosis; chronic renal insufficiency; or issues of substance abuse. A total of 42 patients were enrolled and consented and underwent neurocognitive tests, 1 to 10 days preoperatively. After surgery, patients again underwent neurocognitive testing, 4 days and 30 days postoperatively. Forty percent of patients developed NCD postoperatively. NCD was defined as a $1-\mathrm{cm}$ deviation decline from baseline in $25 \%$ of tasks. This table demonstrates that patients had similar preoperative characteristics in the 2 cohorts, including age, sex, and race, as well as other comorbidities. Similarly, this table demonstrates operative techniques and data that were similar between the groups, including procedure, time, time on CPB, cross-clamp time, and use of cardiotomy suction and cell-saver.

Whole blood was collected preoperatively, 6 hours after CPB, and 4 days after CPB. Immediate mRNA (messenger RNA) extraction and stabilization were done with the whole blood, for microarray analysis. Analysis was performed on samples in duplicate, via blinded analysis. We used the GeneChip HG-U133 Plus 2.0 (Affymetrix Inc, Santa Clara, Calif), which allows for the identification of more than 40,000 genes. We previously validated the gene expression in this group with real-time polymerase chain reaction for a subset of the genes. We used strict discovery criteria and filtering, with the robust multichip average method and a post hoc false-detection rate of $0.05 \%$ to minimize false positives and correct for multiple-comparison testing.

We directly compared gene expression in patients who had NCD (in the blue circle), with those without, whom we labeled "NORM" (in the red circle), at 1 of the 3 time points I mentioned-preoperatively, 6 hours post-CPB, and 4 days postCPB. Of more than 40,000 genes identified on microarray analysis that these 2 groups had in common, there were 140 genes that were significantly differentially regulated between the NORM and NCD groups preoperatively. At 6 hours post-CPB, only 64 genes were differentially regulated, and at 4 days post-CPB, only 25 genes were differentially regulated.

Pathway analysis identified many potential networks that were regulated preoperatively. I will focus on 3 in particular, where several genes were regulated in a similar fashion, meeting bioinformatics threshold criteria. These criteria were inflammation, cell death, and NCD. Preoperatively, several genes associated with T-cell activation and signaling were differentially expressed and were higher in patients who developed NCD postoperatively. The boxes on this graph represent the means; the lines above and below represent the upper and lower confidence intervals. This differential gene expression is of particular interest, as prior studies from our group and others have demonstrated that patients who develop NCD exhibited elevations in their postoperative inflammatory cytokines. Perhaps these genetic differences result in accentuated inflammatory responses and resultant increased chemokine production postoperatively, which we have shown.

Similarly, there was an up-regulation in several genes associated with cell death and oxidative stress. Although these genes are not directly related to one another in a single pathway, they are often indirectly related, and they contribute to downstream inflammatory responses. Other studies have shown that these genes can contribute to impairments in the blood-brain barrier and result in cerebral edema. Perhaps our most interesting finding is the increased expression of several genes implicated in neurologic dysfunction in patients who later did develop NCD.

I would like to take a minute to specifically mention some of what is currently known about these genes. Syncline alpha is a 
major component of amyloid plaques and Alzheimer disease. The FTO or the fat mass and obesity-associated gene has been shown to be inversely related to brain volume and is associated with Alzheimer disease and reduced verbal fluency, specifically in obese patients. TUBB2A is involved in microtubule and axonal guidance. YY transcription factor 1 has many roles in neuronal development and dysfunction and often plays larger roles in activating or repressing gene expression. This slide is a summary of our results. We discussed several genes and their implicated pathways. Again, all of these genes were significantly overexpressed preoperatively in patients who developed NCD, when compared to normal patients. We had a change cutoff of at least 1.5 -fold, and as you see, many of these genes far exceeded that cutoff.

There are limitations to this study. To name a few-foremost, there are a limited number of subjects, as this is a single-institution trial, and although we did have adequate statistical power, it is important to study genetic variations in a larger sample of patients. One of the limitations of any microarray study is the error of "multiple-comparison" testing. Given that tens of thousands of genes are being investigated, it is important to apply stringent sampling filters and statistical corrections. We performed ANOVA (analysis of variance) testing with multiple-comparison corrections and strict filters to prevent these errors. This sort of error possibility is always something that we must be cautious of in these types of studies, and a critical next step is to confirm these findings in a new cohort of patients using prospective testing of these genes.

In summary, this work represents the first study to directly investigate differences in gene expression in patients with and without NCD pre- and post-CPB. The findings suggest that patients who develop NCD are most likely to have preoperative differences in gene expression that may make them more likely to develop NCD after CPB. These studies should serve as a database to guide further investigation and new randomized studies, prospectively testing whether these genes indeed are predictive of NCD. Ultimately, the goal of these studies is to improve preoperative patient selection and individualized therapeutic techniques.

I would like to thank you all for the opportunity to present, again, and I would like to thank my coauthors and Dr Sellke for his mentorship.

Dr Chuong Hoang (Stanford, Calif). I want to congratulate you on a great talk. Dr Sabe and colleagues today have presented to us an intriguing study of gene expression profiles derived from blood samples of patients undergoing bypass procedures. By comparing the gene signatures from presurgical blood samples, they identified differentially expressed genes in patients who subsequently developed neurocognitive decline. This is a highly complex area of investigation - to find these genes that may reflect brain dysfunction. Your efforts thus far are intriguing and, I think, warrant more investigation. I also thank you for sending me the manuscript in advance to review. In this context, I have 2 areas of broad questions to pose to you. The first is: "What is the specific selection and verification process you use to find these gene signatures in blood that are associated with brain dysfunction?" Typically, when conducting gene expression profiling, the disease organ or tissue itself is assessed directly, not necessarily the blood, which is a surrogate compartment. In your study, the gene signatures are derived from cells in the bloodstream. Do these cells actually reflect the status of the brain accurately?
The second area of questioning relates to the number of differentially expressed genes, which was actually the lowest at the 4day time point when neurocognitive decline was measured and present. This observation seems counterintuitive, because one would expect the highest number of genes to be altered during the disease state, when it is active, and not necessarily beforehand, preoperatively. Have you explored this phenomenon in more depth? Those are my questions.

I thank our Association for the privilege of being able to review this paper.

Dr Sabe. Thank you so much for your comments and questions. To address the first question: It definitely is a limitation that we measured gene expression in blood. Obviously, we cannot biopsy brain tissue in these patients, which would give us, possibly, the best measure. However, given some of the studies from our group and several others, patients who developed NCD have been shown to have increased markers of inflammation, and certainly in all of these studies, the markers measured, including inflammatory cytokines, were all measured in blood, and thus may not be directly regulated in brain tissue alone. We did talk about specific genes associated with neurologic function, but as far as the inflammatory component, I think the blood would provide a fair assessment. We did also look at skeletal muscle, as this has been thought to better approximate gene expression in the brain, but there was not really any correlation between the blood and the skeletal muscle. These findings are included in our article.

I am sorry, could you just remind me what your second question was?

Dr Hoang. It concerns the actual number of genes that were differentially expressed. You found the greatest number during the preoperative period, as opposed to when you were actually measuring the neurocognitive decline.

Dr Sabe. Right. That is an excellent question. This has been, over the past 15 to 20 years, a very difficult problem. We have changed many intraoperative and postoperative factors in attempts to decrease the incidence of type II deficits. As I mentioned, this effort has not been fruitful. The only factors that have been shown to be associated with NCD are age, baseline neurologic deficits, depression or psychological deficits, and then inflammation postoperatively, as demonstrated by increased inflammatory cytokines.

So, based on these results, we hypothesize that the preoperative genes, ie, genes that you come in with at baseline, result in type II deficits as seen with NCD. Many of these genes are associated with inflammation downstream. The baseline differential elevation in expression of these genes makes these patients more likely to develop inflammation, or "primes" them preoperatively, so once they undergo the CPB and the stresses of surgery they would be at higher risk of activating the T-cell cascades and antigen presentation, and other inflammatory pathways that would ultimately lead to NCD. After the major stress of surgery, we likely have a "settling out" of all the inflammatory genes that would be regulated, and patients likely have a similar genetic response to this. Thus, as time passes postoperatively, we can possibly explain the decreased number of differentially regulated genes as a function of patients reacting to the same stress, in an overall and expectedly similar manner. Thus, we theorize that the biggest gene differences seen preoperatively have primed patients to develop NCD. That is a long answer, but I hope it answers your question.

Dr Hoang. It does. Thank you very much. 